\title{
Factor XII (Hageman) Levels in Women with Recurrent Pregnancy Loss
}

\author{
A. Seval Ozgu-Erdinc, ${ }^{1}$ Cihan Togrul, ${ }^{2}$ Ayla Aktulay, ${ }^{1}$ Umran Buyukkagnici, ${ }^{3}$ \\ Elif Gul Yapar Eyi, ${ }^{1}$ and Salim Erkaya ${ }^{1}$ \\ ${ }^{1}$ Department of Obstetrics and Gynecology, Zekai Tahir Burak Women's Health Care, Training and Research Hospital, \\ Talatpasa Bulvari, 06230 Ankara, Turkey \\ ${ }^{2}$ Department of Obstetrics and Gynecology, Diyarbakır Women's Health and Children Disease Hospital, Diyarbakır, Turkey \\ ${ }^{3}$ Department of Biochemistry, Zekai Tahir Burak Women's Health Care, Training and Research Hospital, \\ Talatpasa Bulvari, 06230 Ankara, Turkey
}

Correspondence should be addressed to A. Seval Ozgu-Erdinc; sevalerdinc@gmail.com

Received 31 July 2014; Revised 15 October 2014; Accepted 23 October 2014; Published 12 November 2014

Academic Editor: Antonio Farina

Copyright ( 2014 A. Seval Ozgu-Erdinc et al. This is an open access article distributed under the Creative Commons Attribution License, which permits unrestricted use, distribution, and reproduction in any medium, provided the original work is properly cited.

\begin{abstract}
Objective. To evaluate factor XII levels in women with recurrent pregnancy loss (RPL) in a tertiary referral hospital. Methods. Women who were referred to our hospital for two consecutive abortions or three abortions in between 2007 and 2013 were included in this retrospective observational study. Women were further grouped according to factor XII levels, as $<60 \%$ and $\geq 60 \%$. Results. Mean factor XII level was $109.1 \pm 35.7 \%$ (range: 9-200). Ninety-three (7.4\%) women had factor XII levels $<60 \%$. Mean factor XII level was $44.8 \pm 13.1$, and levels ranged between 9 and 60 in this group. Only one woman had factor XII level $<10 \%$. Remaining 1164 (92.6\%) women had factor XII levels $\geq 60 \%$. Mean factor XII level was $114.3 \pm 31.7$, and levels ranged between 60.3 and 200 in this group, while $1015(72.4 \%)$ women had factor XII levels within the normal range $(60 \%-150 \%[100 \%=30 \mu \mathrm{g} / \mathrm{mL}])$. Conclusion . Decreased activity of F-XII was diagnosed in $7.4 \%$ of women with RPL. We concluded factor XII deficiency that might be a rare but significant factor for RPL, and should be evaluated in women who are investigated for recurrent pregnancy loss.
\end{abstract}

\section{Introduction}

Coagulation and fibrinolysis balance plays an important role in sustaining a normal placental function. Thrombosis of spiral arteries and the intervillous space on the maternal side of the placenta can impair adequate placental perfusion. Impairments in uteroplacental circulation may cause fetal loss, fetal growth restriction, and/or preeclampsia.

Coagulation factor XII (F-XII), which is also known as Hageman factor, is a plasma protein and a single-chain zymogen form of F-XIIa with no detectable enzymatic activity [1]. Factor XII is synthesized in the liver with a molecular weight of $80-90 \mathrm{kD}$ and plasma concentrations are about $30 \mathrm{mg} / \mathrm{mL}$ [1]. Factor XII has a major role in the initiation of the intrinsic pathway of blood coagulation. It activates FXI and prekallikrein in the intrinsic pathway of the coagulation cascade. FXII deficiency is rare and may present as an autosomal recessive or acquired disorder. It is generally asymptomatic. On the contrary, excessive F-XII levels may result in venous thrombosis. Previously many studies suggested an association between factor XII (FXII) deficiency and autoantibodies to FXII and recurrent pregnancy losses [2-5]. In this study, we aimed to evaluate the prevalence of FXII deficiency in women who were investigated for recurrent pregnancy loss (RPL) in a tertiary referral hospital.

\section{Methods}

A cross-sectional study was conducted at Zekai Tahir Burak Women's Health Care, Training and Research Hospital after acquiring approval from the institutional review board. Women who were referred to our hospital for two consecutive abortions or three abortions in between 2007-2013 were included in this retrospective observational study. Pregnancy 
TABLE 1: Characteristics of the study population.

\begin{tabular}{lcc}
\hline & & Min-max \\
\hline Age, years (mean \pm SD) & $29.6 \pm 9.2$ & $18-39$ \\
Gravidity (median) & 4 & $1-12$ \\
Pregnancy loss (median) & 2 & $2-10$ \\
Previous live birth (median) & 0 & $0-2$ \\
\hline
\end{tabular}

TABLE 2: Distribution of factor XII levels among patients with recurrent pregnancy loss.

\begin{tabular}{lccc}
\hline & $n$ & $\%$ & Mean \pm SD \\
\hline$<10 \%$ & 1 & 0,1 & 9 \\
$10-35 \%$ & 20 & 1,6 & $25,85 \pm 6,39$ \\
$35-60 \%$ & 73 & 5,8 & $50,81 \pm 7,73$ \\
$60-150 \%$ & 1014 & 80,7 & $105,53 \pm 22,38$ \\
$150-200 \%$ & 149 & 11,9 & $174,50 \pm 17,47$ \\
\hline Total & 1257 & 100 & $109,19 \pm 35,74$ \\
\hline
\end{tabular}

losses after 12 weeks of gestation were not included in the study. Cases in which F-XII levels could not be measured were excluded. Factor XII activity was measured by the APTT method using a Behring Coagulation System (BCS; Dade Behring Inc., Liederbach, Germany). FXII-deficient plasma (George King Bio-Medical Inc., St. Overland Park, KS, USA) was incubated with Pathromtin SL (Dade Behring Inc.) and $0.02 \mathrm{~mol} / \mathrm{L} \mathrm{CaCl}_{2}$ (Calcium Chloride) (Sysmex International Reagents Co., Ltd., Kobe, Japan). The mixture was incubated with patient plasma or standard plasma and the clotting time was recorded. Activity was expressed as a percentage with reference to the curve for standard human plasma. Women were further grouped according to F-XII cut-off levels 10, 35, 60 , and $150[3,6]$. Statistical analyses were performed with SPSS for Windows 21.0 software. Continuous variables were expressed as mean \pm standard deviation (SD), and categorical variables were expressed as number (percentage).

\section{Results}

Within the study period, 1401 women were assessed for FXII levels. Among these, 144 women were excluded because of technical unavailability of F-XII measurements. A total of 1257 women were eligible for final analysis. Mean patient age was $29.6 \pm 9.2$ years (range 18-39). Median abortion count was 2 (range 2-4) (Table 1). Mean F-XII level was $109.1 \pm$ $35.7 \%$ (range 9-200) (Table 2). Ninety-three (7.4\%) women had F-XII levels $<60 \%$. Mean F-XII level was $44.8 \pm 13.1$, and levels ranged between 9 and 60 in this group. Only 1 woman had F-XII level $<10 \%$ and 20 women had F-XII levels $<35 \%$ which makes the F-XII deficiency prevalence of our study population 1.7\%. Remaining 1164 (92.6\%) women had F-XII levels $\geq 60 \%$. Mean F-XII level was $114.3 \pm 31.7$, and levels ranged between 60.3 and 200 in this group.

\section{Discussion}

Recurrent pregnancy loss may be associated with disturbances in coagulation and fibrinolysis cascade. In a recent study from Greece, FXII deficiency and the number of abortions in the recurrent abortion group were found to be correlated [6]. In previous studies the prevalence of FXII deficiency has been reported between 2.9 and $15 \%$ of women with RPL [3, 6-8]. Also in Matsubayashi et al's study [9], decreased activity of F-XII was found to be associated with recurrent in vitro fertilization failure. There have been previous reports of an association of FXII deficiency with RPL [2-5] and in a larger study of 500 women with unexplained primary recurrent miscarriages [10]; F-XII deficiency and hypofibrinolysis were found to be the most frequent abnormalities of the hemostatic system [11].

The presence of antibodies to factor XII has been shown to lead to F-XII deficiency [4]. In the routine setting neither factor XII levels nor antibodies against factor XII are evaluated. The etiology could not be explained in the half of patients with recurrent pregnancy loss. Presence of antibodies to factor XII and factor XII deficiency may help to reveal a cause for unexplained RPL.

In this study, we investigated F-XII levels in women with RPL. In our study 7.4\% of women with RPL had reduced F-XII activity, while F-XII deficiency was present in only $1.7 \%$. Therefore, we conclude that although FXII deficiency might be a rare but significant factor for RPL, and should be evaluated in women who are investigated for recurrent pregnancy loss.

\section{Conflict of Interests}

The authors report no conflict of interests. The authors alone are responsible for the content and writing of the paper.

\section{Acknowledgment}

This paper was selected as a poster presentation in The World Congress on Building Consensus out of Controversies in Gynecology, Infertility and Perinatology (BCGIP-COGI), Istanbul, May 30-June 2, 2013.

\section{References}

[1] T. Sugi, "Kininogen-dependent antiphosphatidylethanolamine antibodies and autoantibodies to factor XII in patients with recurrent pregnancy losses," Journal of Obstetrics and Gynaecology Research, vol. 39, no. 7, pp. 1223-1229, 2013.

[2] A. Inomo, T. Sugi, Y. Fujita, H. Matsubayashi, S.-I. Izumi, and M. Mikami, "The antigenic binding sites of autoantibodies to factor XII in patients with recurrent pregnancy losses," Thrombosis \& Haemostasis, vol. 99, no. 2, pp. 316-323, 2008.

[3] H.-U. Pauer, P. Burfeind, H. Köstering, G. Emons, and B. Hinney, "Factor XII deficiency is strongly associated with primary recurrent abortions," Fertility and Sterility, vol. 80, no. 3, pp. 590-594, 2003.

[4] D. W. Jones, M. J. Gallimore, and M. Winter, "Antibodies to factor XII: a possible predictive marker for recurrent foetal loss," Immunobiology, vol. 207, no. 1, pp. 43-46, 2003.

[5] A. Sotiriadis, A. Makrigiannakis, T. Stefos, E. Paraskevaidis, and S. N. Kalantaridou, "Fibrinolytic defects and recurrent 
miscarriage: a systematic review and meta-analysis," Obstetrics and Gynecology, vol. 109, no. 5, pp. 1146-1155, 2007.

[6] S. Dendrinos, A. Deliveliotou, A. Anastasiou, and G. K. Creatsas, "Role of coagulation factor XII in unexplained recurrent abortions in the Greek population," Journal of Reproductive Medicine, vol. 59, no. 1-2, pp. 56-62, 2014.

[7] H. Yamada, E. H. Kato, G. Kobashi et al., "Recurrent pregnancy loss: etiology of thrombophilia," Seminars in Thrombosis and Hemostasis, vol. 27, no. 2, pp. 121-129, 2001.

[8] H. Yamada, E. H. Kato, Y. Ebina et al., "Factor XII deficiency in women with recurrent miscarriage," Gynecologic and Obstetric Investigation, vol. 49, no. 2, pp. 80-83, 2000.

[9] H. Matsubayashi, T. Sugi, T. Suzuki et al., "Decreased factor XII activity is associated with recurrent IVF-ET failure," American Journal of Reproductive Immunology, vol. 59, no. 4, pp. 316-322, 2008.

[10] I. Braulke, M. Pruggmayer, P. Melloh, B. Hinney, H. Kostering, and E. Gunther, "Factor XII (Hageman) deficiency in women with habitual abortion: new subpopulation of recurrent aborters?" Fertility and Sterility, vol. 59, no. 1, pp. 98-101, 1993.

[11] J.-C. Gris, S. Ripart-Neveu, C. Maugard et al., "Prospective evaluation of the prevalence of haemostasis abnormalities in unexplained primary early recurrent miscarriages," Thrombosis and Haemostasis, vol. 77, no. 6, pp. 1096-1103, 1997. 


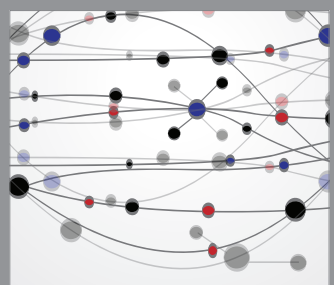

The Scientific World Journal
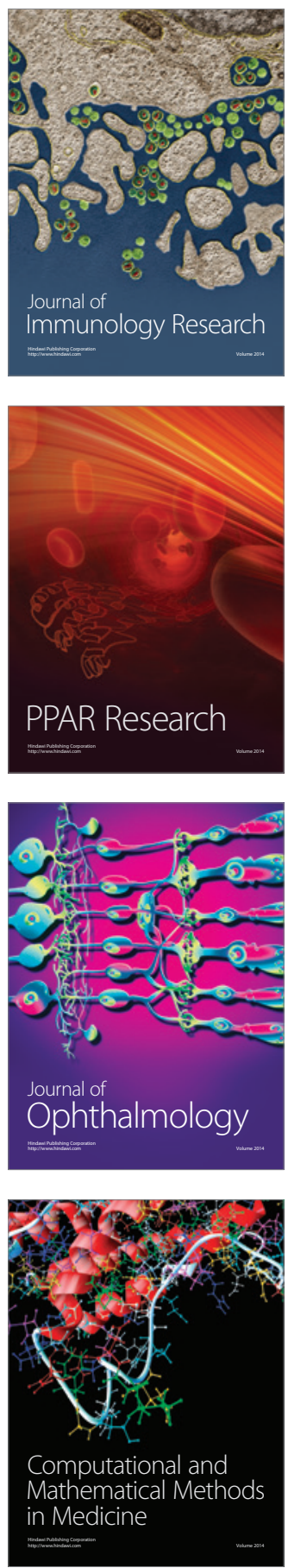

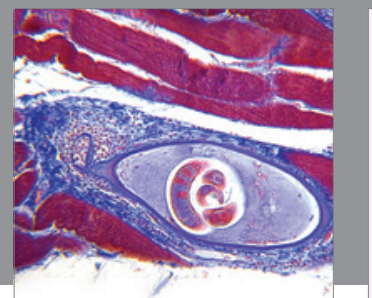

Gastroenterology

Research and Practice
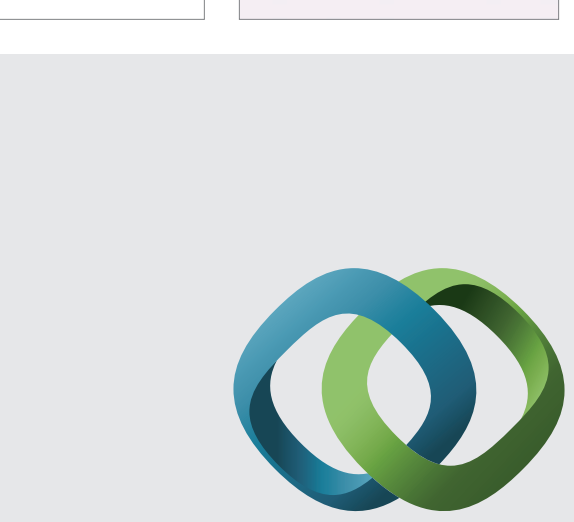

\section{Hindawi}

Submit your manuscripts at

http://www.hindawi.com
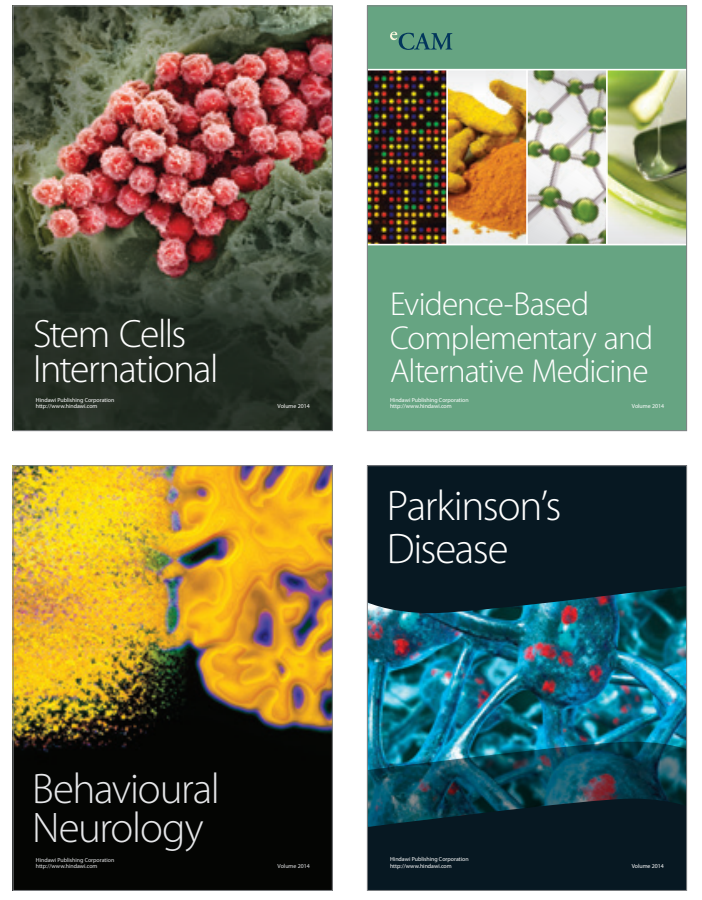
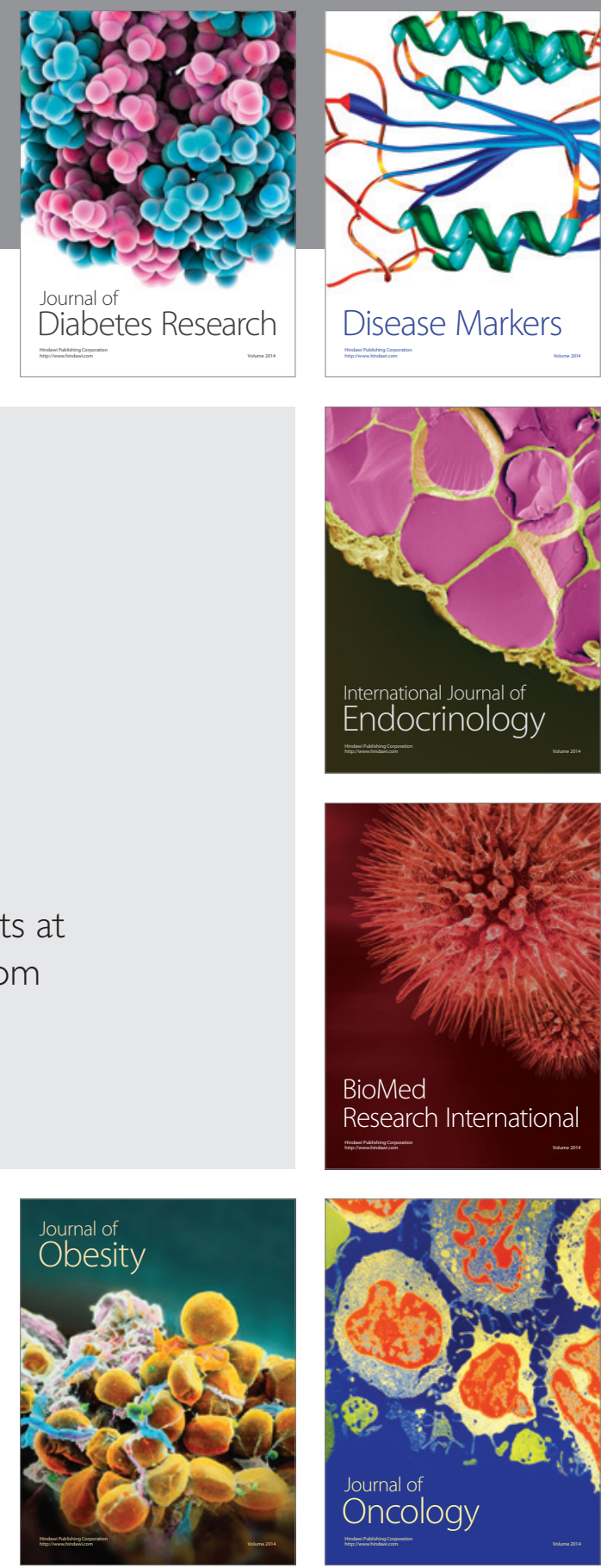

Disease Markers
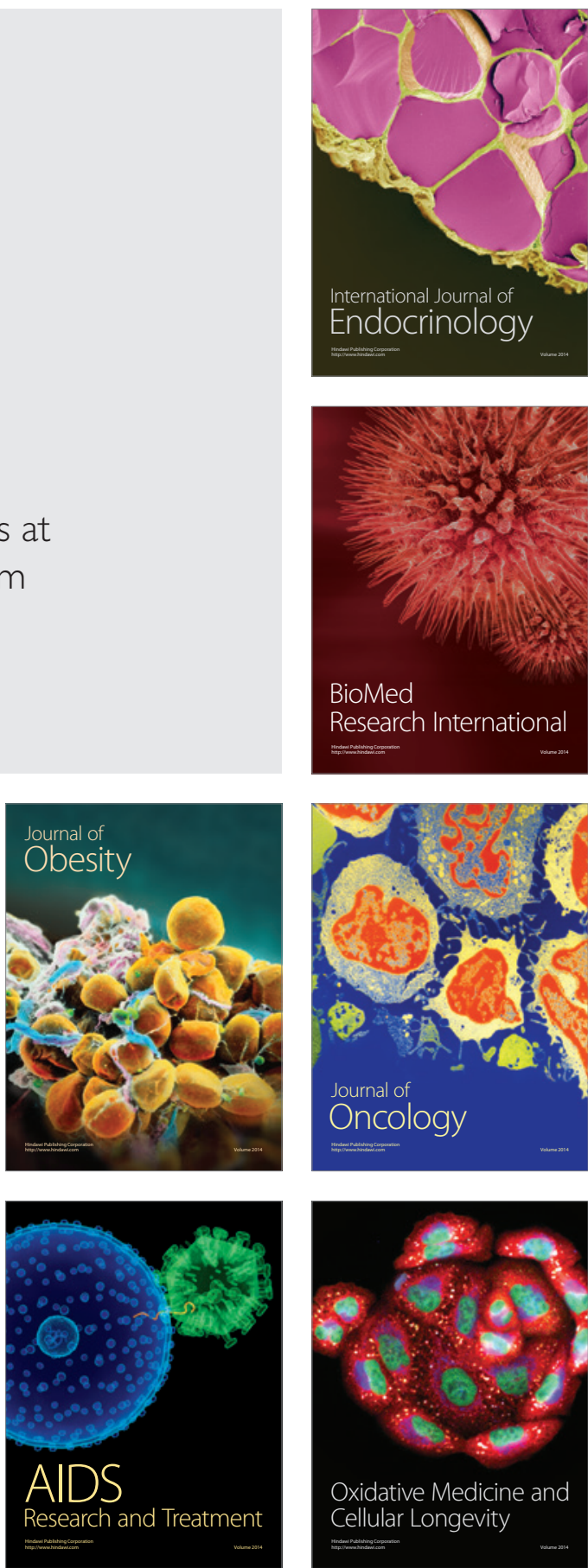